\title{
Simulation of the misalignment process of an overhead crane in Matlab / Simulink
}

\author{
Alexandr Komarov ${ }^{1}$, Alexey Grachev ${ }^{1}$, Anton Gabriel ${ }^{1}$ and Natalya Mokhova ${ }^{1}$ \\ ${ }^{1}$ Institute of Machinery, Materials, and Transport, Peter the Great St. Petersburg Polytechnic \\ University, 195251 St. Petersburg, Russia
}

\begin{abstract}
The object of the study is the angle of deviation of the crane from the perpendicular to the rails in a horizontal plane. The aim of the work is the mathematical description of the crane's bridge beam misalignment, creation of the model in Matlab Simulink. Based on the resulting model, there was implemented a control system that compensates for emerging misalignment by speeding up or slowing down crane drives.
\end{abstract}

\section{Introduction}

The problem of the frame's misalignment on the horizontal plane is relevant for all types of overhead cranes. There are different reasons for the misalignment: uneven abrasion of wheels, the roughness of the crane track (crane rails). The main parameter for the misalignment emerging is the mass center of system crane-load shift relative to the crane's center of symmetry. That results in the crane's drives uneven loading which leads to running of one support relative to another - misalignment.

Usually, this problem is not fixed by the manufacturer. It is known as a common issue with such types of cranes. However, the misalignment causes the occurrence of additional stresses in a crane's construction and the installation structure. Uneven parts production, a decrease in the operation period, and even crane collapse can be the result of the misalignment of the crane's moving.

In the article [1] the usage of the additional adjusting control system was proposed for receiving the crane operator's control signals, adjustment of them, and generating a new control action on the drives. From the review of the existing sensors and their installation for detecting the misalignment, it was determined that a laser ranging device is best used as a sensor.

In the following article, it is considered a mathematical description of the crane's misalignment process and the development of a simple control system for fixing the misalignment.

\section{Mathematical Description}

It is needed to create a crane's mathematical model which will meet the required parameters to implement a model of the control system in Matlab. To select a modeling object the overhead crane market was reviewed to find a manufacturer which provides detailed technical specification of the crane in open access. Stahl Crane Systems company 
was selected because of the review. A crane with the following technical parameters was selected as an example for the modeling [2].

Table 1. Main technical characteristics of the crane

\begin{tabular}{|c|c|}
\hline Load capacity & $16000 \mathrm{~kg}$ \\
\hline Crane weight & $4300 \mathrm{~kg}$ \\
\hline Crane span length & $15000 \mathrm{~mm}$ \\
\hline Max speed & $40 \mathrm{~m} / \mathrm{min}$ \\
\hline Wheels' diameter & $315 \mathrm{~mm}$ \\
\hline
\end{tabular}

More detailed specifications are described in the company's catalog. Based on the technical specifications provided by the manufacturer and overhead cranes' design features [3][4] there was defined and selected all parameters for the creation of the required crane mathematical model such as the wheels' width and bearing rib, crane rails type and dimensions. The block diagram of the created model is shown in Fig. 1.

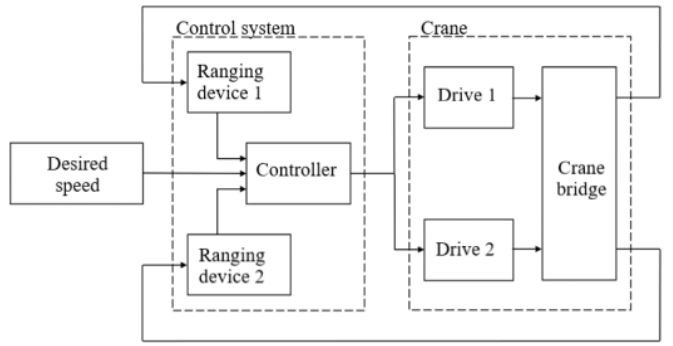

Fig. 1. Scheme of the mathematical description problem

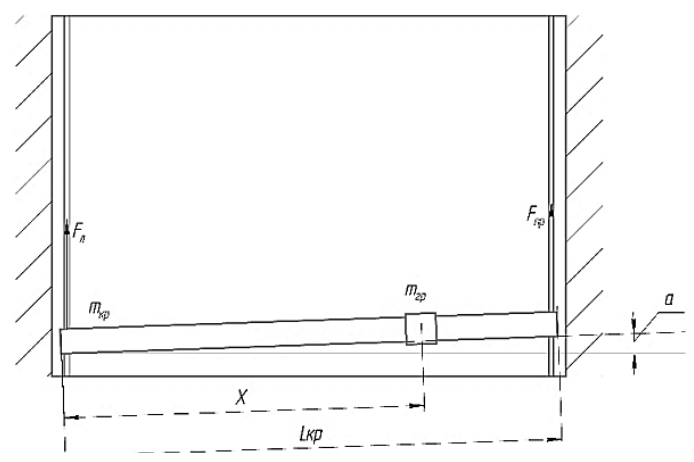

Fig. 2. Scheme of the mathematical description problem

A search for scientific articles on this topic has revealed that most publications are about descriptions and suggestions for solution to load swinging during transportation. But there are not so much researches about the topic of the current work. However, the authors [5][6] consider the problem of the misalignment of metal structures of overhead cranes from the point of view of stresses arising in the structure. Also, a method of mathematical description of load swinging can be used for the current problem [7][8][9]. A mathematical model is described by Euler-Lagrange formulas of the second kind, in which the Lagrangian operator is a difference between total kinetic energy and total potential energy 
of the system. Crane's $x$ coordinate and angle of deviation of the crane from the perpendicular to the motion vector are used as generalized coordinates. The scheme of the considered problem is shown in Fig. 2.

The potential energy of the system can be neglected in the current problem. Then the Lagrangian operator is equal to the total kinetic energy of the system that consists of the kinetic energy of the rectilinear motion of the crane and the load, the kinetic energy of crane's rotation, and kinetic energy of the payload:

$$
T=\frac{m \dot{x}^{2}}{2}+\frac{J_{c r} \dot{\alpha}^{2}}{2}+\frac{J_{p l} \dot{\alpha}^{2}}{2}
$$

Where $m$ - the sum of the crane mass and the payload mass;

$J_{c r}-$ moment of inertia of the crane rotation;

$J_{p l}$ - moment of inertia of the payload rotation.

To find moments of inertia for the crane and the payload, it is needed to know the coordinates of the mass center, which depends on the position of the payload mass on the crane:

$$
C M=\frac{L_{c r}}{2}+\frac{X-\frac{L_{c r}}{2}}{1+\frac{m_{c r}}{m_{p l}}}
$$

Where $L_{c r}$ - length of the crane span;

$X$ - the position of the payload relative to the left edge of the crane;

$m_{c r}$ - crane mass;

$m_{p l}$ - payload mass.

The moment of inertia of the crane rotation relative to the mass center can be described as a sum of two moments of inertia of two rods rotating around the axis of rotation. The payload moment of inertia is described as a rotation of a material point around the axis of rotation.

As a result, the formula of the total kinetic energy has the following form:

$$
T=\frac{\left(m_{c r}+m_{p l}\right) \dot{x}^{2}}{2}+\frac{m_{c r}\left(2 \frac{C M^{2}}{L_{C r}}+L_{c r}{ }^{2}+C M^{2}-3 L_{c r} C M\right) \dot{\alpha}^{2}}{6}+\frac{m_{p l}(C M-X)^{2} \dot{\alpha}^{2}}{2}
$$

The traction force of the drives acting on the crane can be found by newton's second law. Thereby, the generalized force for the $x$ coordinate is presented in the formula (4).

$$
Q_{1}=R_{w} \cos \alpha\left(\left(\frac{m_{c r}}{2}+m_{p l} \frac{L_{c r}-C M}{L_{c r}}\right) \frac{d \omega_{1}}{d t}+\left(\frac{m_{c r}}{2}+m_{p l} \frac{C M}{L_{c r}}\right) \frac{d \omega_{2}}{d t}\right)
$$

Unequal values of the torques of the drives directed towards each other affect the crane rotation. The generalized formula for the $\alpha$ coordinate is presented in the formula (5).

$$
Q_{2}=R_{w}\left(C M\left(\frac{m_{c r}}{2}+m_{p l} \frac{L_{c r}-C M}{L_{c r}}\right) \frac{d \omega_{1}}{d t}-\left(L_{c r}-C M\right)\left(\frac{m_{c r}}{2}+m_{p l} \frac{C M}{L_{c r}}\right) \frac{d \omega_{2}}{d t}\right)
$$

Where $R_{w}$-wheel radius.

In the current work, the friction force of the wheel edges on the rails is not considered as if the control system operates correctly and there is done a proper maintaining of the crane rails and the crane those forces will be random and short. So, there is no need to model 
them. Substituting the obtained equations 3, 4, 5 into the Euler-Lagrange formula of the second kind, we get:

$$
\left\{\begin{array}{c}
\ddot{x}=\frac{R_{w} \cos \alpha\left(\left(\frac{m_{c r}}{2}+m_{p l} \frac{L_{c r}-C M}{L_{c r}}\right) \frac{d \omega_{1}}{d t}+\left(\frac{m_{c r}}{2}+m_{p l} \frac{C M}{L_{c r}}\right) \frac{d \omega_{2}}{d t}\right)}{\left(m_{c r}+m_{p l}\right)} \\
\ddot{\alpha}=\frac{3 R_{w}\left(C M\left(\frac{m_{c r}}{2}+m_{p l} \frac{L_{c r}-C M}{L_{c r}}\right) \frac{d \omega_{1}}{d t}+\left(L_{c r}-C M\right)\left(\frac{m_{c r}}{2}+m_{p l} \frac{C M}{L_{c r}}\right) \frac{d \omega_{2}}{d t}\right)}{\left(m_{c r}\left(2 \frac{C M^{3}}{L_{c r}}+L_{c r}{ }^{2}+C M^{2}-3 L_{c r} C M\right)+3 m_{p l}(C M-X)^{2}\right)}
\end{array}\right.
$$

One of the main reasons for the crane misalignment occurrence is the dependence of the drive rotation speed on the applied load torque. Maximum driver speed must be limited depending on the coordinate of the payload position and its mass while modeling in Matlab. In the work [10] it is described a detailed model of the crane drives which is redundant in this paper. In further work it is planned to make a more realistic model. As drives in the crane model under study are used with frequency converter, such drive can be considered as a direct current motor with a rigid mechanical characteristic from the point of view of the control system and mechanical characteristic.

Table 2. АИР63В4 motor parameters

\begin{tabular}{|c|c|c|}
\hline Parameter & Marking & Value \\
\hline Power, $\mathrm{kW}$ & $\mathrm{P}$ & 0,33 \\
\hline Power factor & $K_{p}$ & 0,76 \\
\hline $\begin{array}{c}\text { The ratio of the maximum moment to } \\
\text { the minimum }\end{array}$ & $h$ & 2,2 \\
\hline Synchronous speed, RPM & $n_{0}$ & 1500 \\
\hline Speed at nominal torque, RPM & $n_{n}$ & 1325 \\
\hline
\end{tabular}

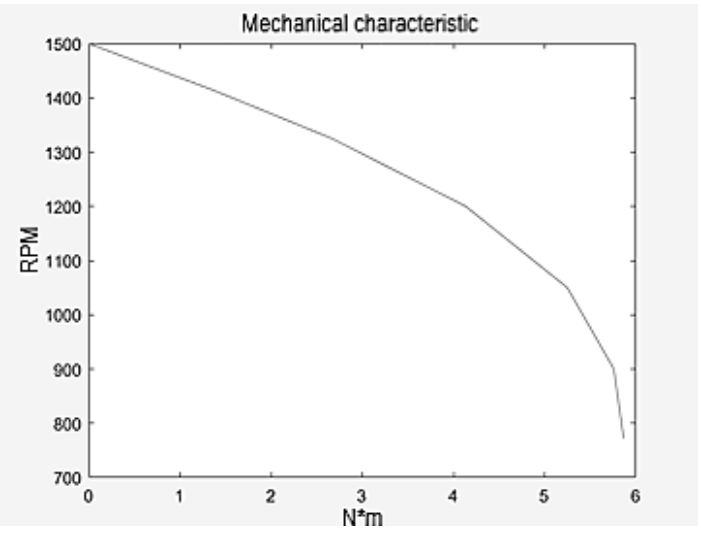

Fig. 3. Section of the mechanical characteristic

Direct current motor is described using second-order aperiodic link consisting of two consecutive first-order aperiodic links which describe electromagnetic and mechanical characteristics, respectively. Drive electromagnetic characteristics with vector control might not be considered as it is extremely difficult to find electromagnetic characteristics for the crane's drive. Thus, the engine model is reduced to a first-order aperiodic link with PID control and negative inverse connection on speed.

In the Stahl Crane Systems catalog [11] for crane drives, not enough parameters are provided to determine drive mechanical characteristics. An analog of the Russian production with similar basic parameters was selected but its parameters were also 
insufficient to determine the mechanical characteristic. Cage asynchronous motor АИР63B4 was selected. It is necessary to design a mechanical characteristic for the selected motor to implement in the model the dependence of the maximum drive speed and the load torque generated by the motor. АИР63В4 motor parameters are shown in table 2. The section of mechanical characteristics of interest is constructed for the selected motor, as shown in Fig. 3.

Speed range from $1500 \mathrm{rpm}$ to $1000 \mathrm{rpm}$ can be considered linear and can be described by the equation of a straight line with a slope coefficient $k_{n}=77,6$. Thus, the maximum speed of the motor is described by the formula (7).

$$
\omega_{\max }=n_{0}-\frac{M_{n} Z}{M_{r} k_{n}}
$$

Where $Z$-gear reduction rate;

$M_{n}$ - nominal torque;

$M_{r}$ - moment of resistance (load) acting on the drive.

The authors [12] have obtained a formula (8) for calculating the moment of resistance acting on the drives.

$$
M_{c}=(Q+G)\left(k+\frac{f d}{2}\right) k_{p}
$$

Where $Q, G$ - crane and load masses, respectively, measured in tons;

$k$ - rolling friction coefficient of the steel wheel on the rail;

$f$ - reduced coefficient of friction in bearings;

$d$ - axle diameter;

$k_{p}$ - coefficient of friction of the rib.

The coefficient $k_{p}$ is assumed to be equal to one as the friction force of the wheels on the edges is not taken into consideration.

As the control system is working with the step of the discreteness of ranging devices used to measure the covered distance and drives' speed is needed to be controlled, it is necessary to implement the numerical differentiation block. The system will get the speed of the movement from the movement function due to the block. As a method of numerical differentiation, the finite difference method on a three-point scheme is used.

\section{Matlab/Simulink Implementation}

Fig. 4 shows the final crane drive model developed in Matlab Simulink. For ease of use of the drive model, the linear speed of the movement is used as input and output signals, and not the speed of rotation of the motor itself. The maximum speeds depending on the load torque calculated in the previous part are specified as the parameters of the restrictions in the saturation block.

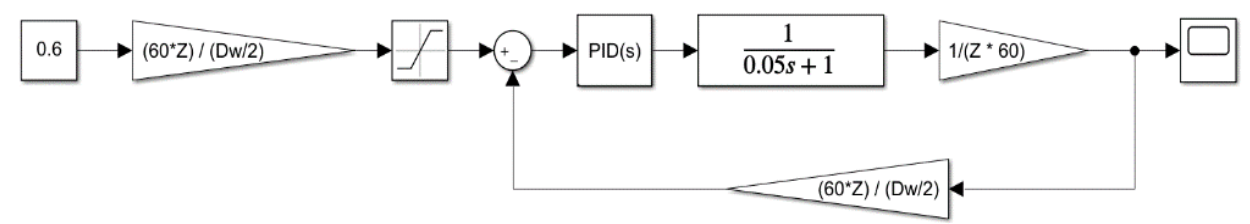

Fig. 4. Drive model in Matlab Simulink 


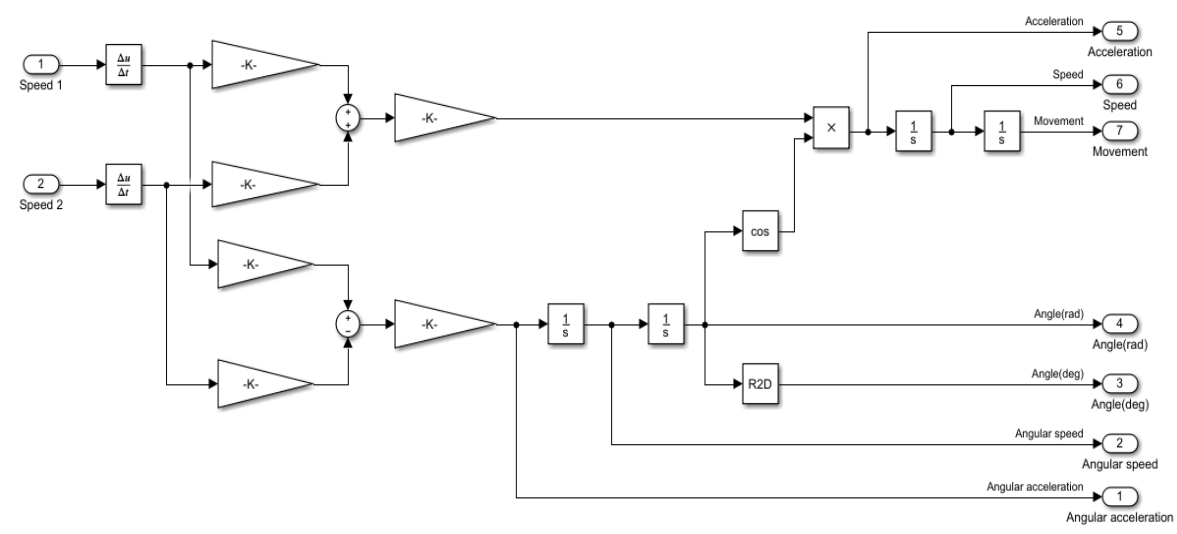

Fig. 5. Crane model in Matlab Simulink

Also, Fig. 6 shows a block that calculates the coordinates of the left and right parts of the crane, which will be the output coordinates of the entire crane model. The final crane model is shown in Fig. 7.

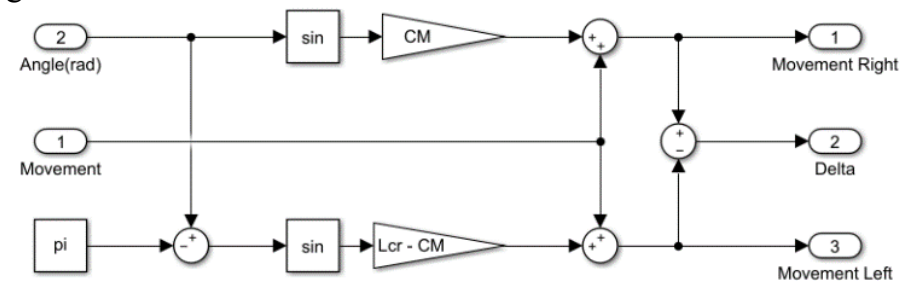

Fig. 6. Block for calculating the coordinates of the right and left parts of the crane.

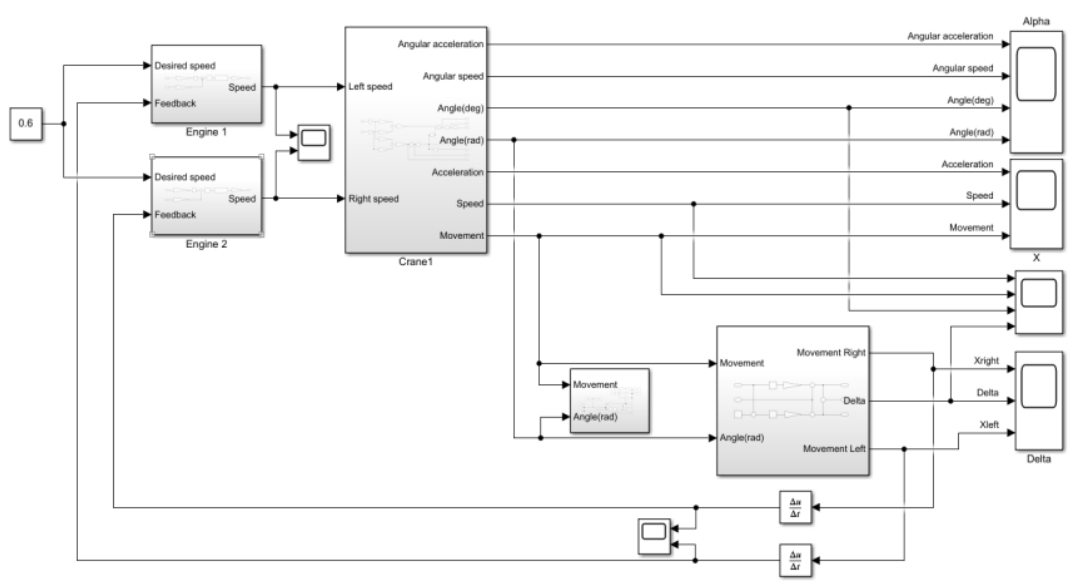

Fig. 7. Final crane model in Matlab Simulink

The final model of the crane developed in Matlab Simulink is shown in Fig. 5. The linear speeds of the left and right drives are fed to the input of the system, respectively. Then these values are differentiated to obtain the values of the accelerations of the left and right parts of the crane. Parts of the equations from formula (6) are written in the amplification blocks. After the amplification blocks, the values of linear acceleration (in the upper part of the diagram) and angular acceleration of the crane rotation (in the lower part of the diagram) are displayed. Using integration blocks, the final output parameters of the model are: 
- linear acceleration of the center of mass;

- linear speed of the center of mass;

- coordinate of the center of mass position;

- angular acceleration of crane rotation around the center of mass;

- the angular speed of the crane rotation around the center of mass;

- the angle of deviation of the crane from the motion vector in radians;

- the angle of deviation of the crane from the motion vector in degrees.

First of all, the control system must contain sensors that will ensure the availability of upto-date information about the position of the crane in space. Within the framework of work [1], a pair of laser rangefinders were selected as sensors. Also, studies have shown that laser range finders are the least susceptible to changing environmental conditions and it is more expedient to use them as sensors of the system [13-15].

The crane model, created in Matlab Simulink, outputs the positions of the right and left parts of the crane as output signals. This already performs the functionality of rangefinders, but it is important to take into account that the rangefinder signal is not constant, and the data on the position of the crane is discrete. It is also important to note that rangefinders have some measurement error, and sometimes noise and interference are superimposed on the measurements. In this paper, the noise power applied to the rangefinder signal is assumed to be zero, since noise filtering requires a separate review and selection of filters. Thus, the rangefinder model is reduced to a signal sampling block and adding white noise to this signal. At the input, such a module takes the position of one of the sides of the crane, obtained from a previously made model, and at the output, the distance measured by rangefinders, which is close to the real one. The resulting diagram of the rangefinder block is shown in Fig. 8.

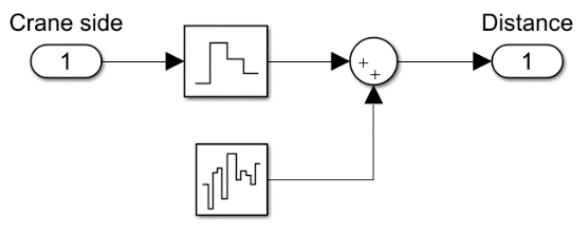

Fig. 8. Model of a laser rangefinder in Matlab Simulink

A PI regulator is used as a regulator of the control system. The difference between the readings of the laser rangefinders is fed to the input of the regulator and the regulator reduces this difference to zero. The output of the regulator is connected to the previously created numerical differentiation block to obtain the value of the correction speeds for the drives.

The model of the control system in Matlab Simulink is shown in Fig. 9. Fig. 10 shows a model of the entire system. It is a block of the crane model described earlier, two laser rangefinder modules and a control system block.

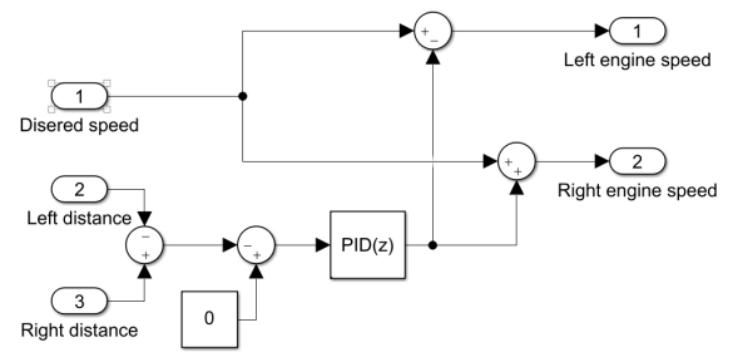

Fig. 9. Control system model in Matlab Simulink 


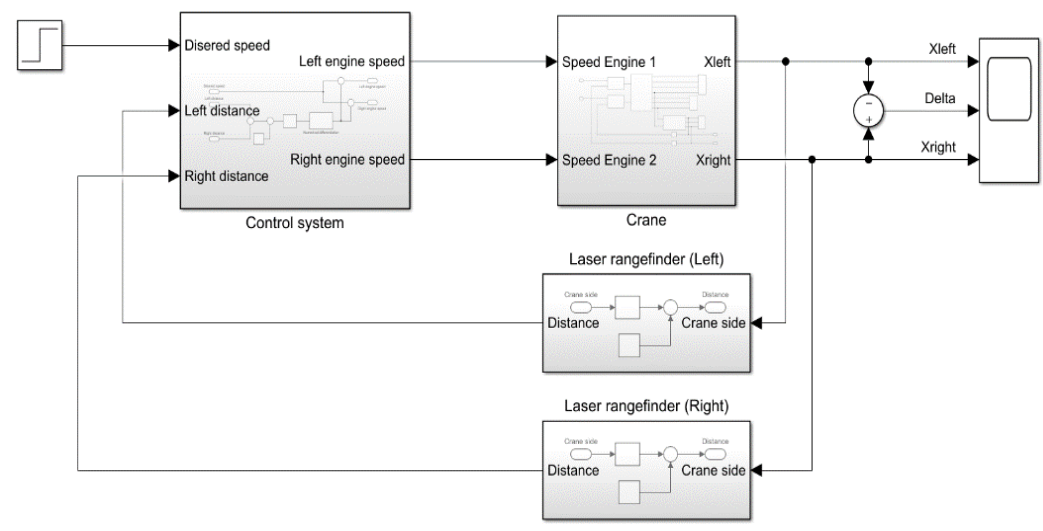

Fig. 10. Complete system model

\section{Experiment in Matlab/Simulink}

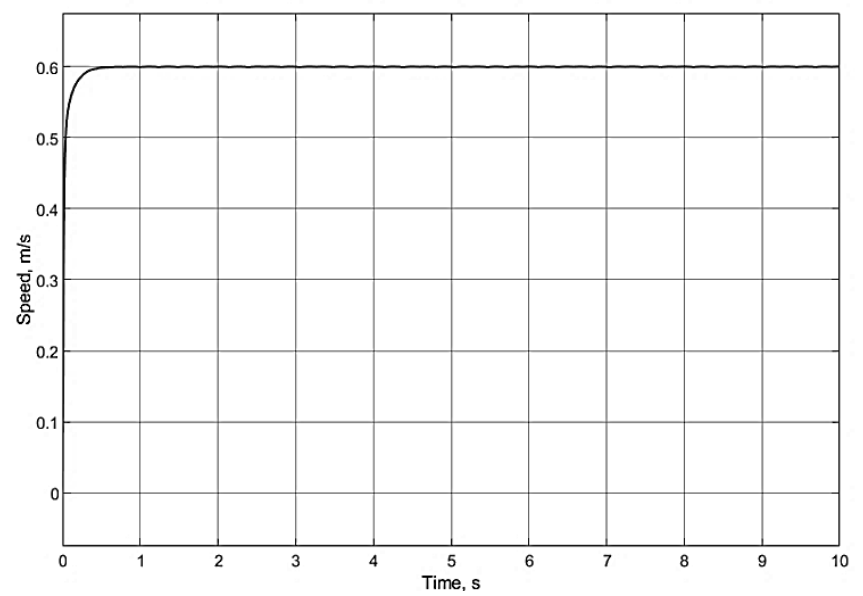

Fig. 11. Graph of the transition process in the drive model
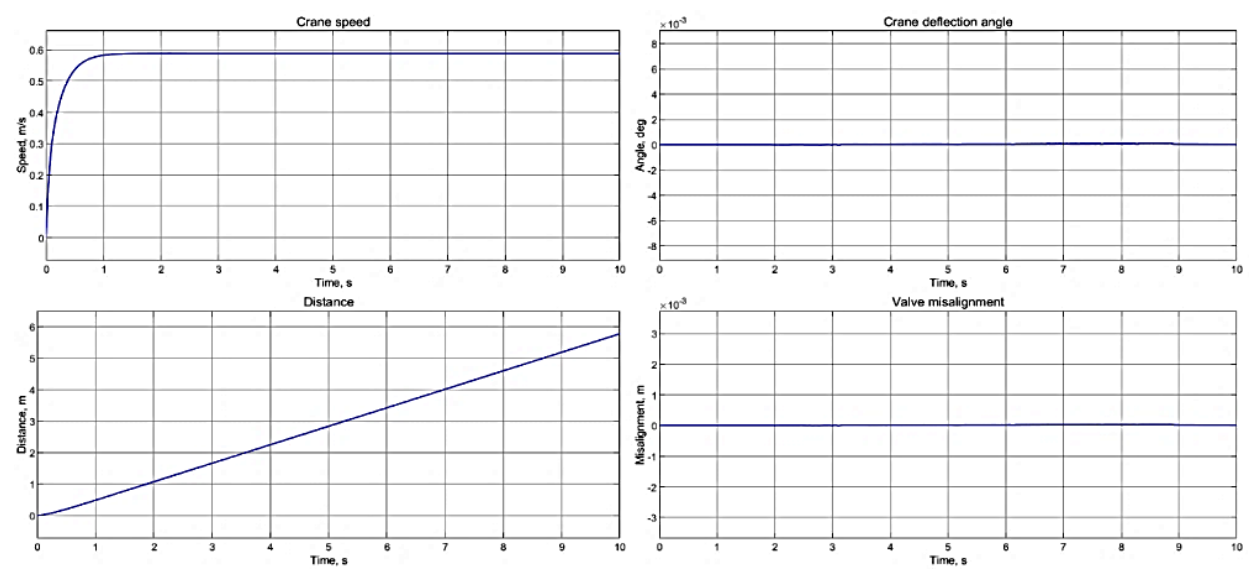

Fig. 12. Graphs of model parameters with a load in the center of the crane 

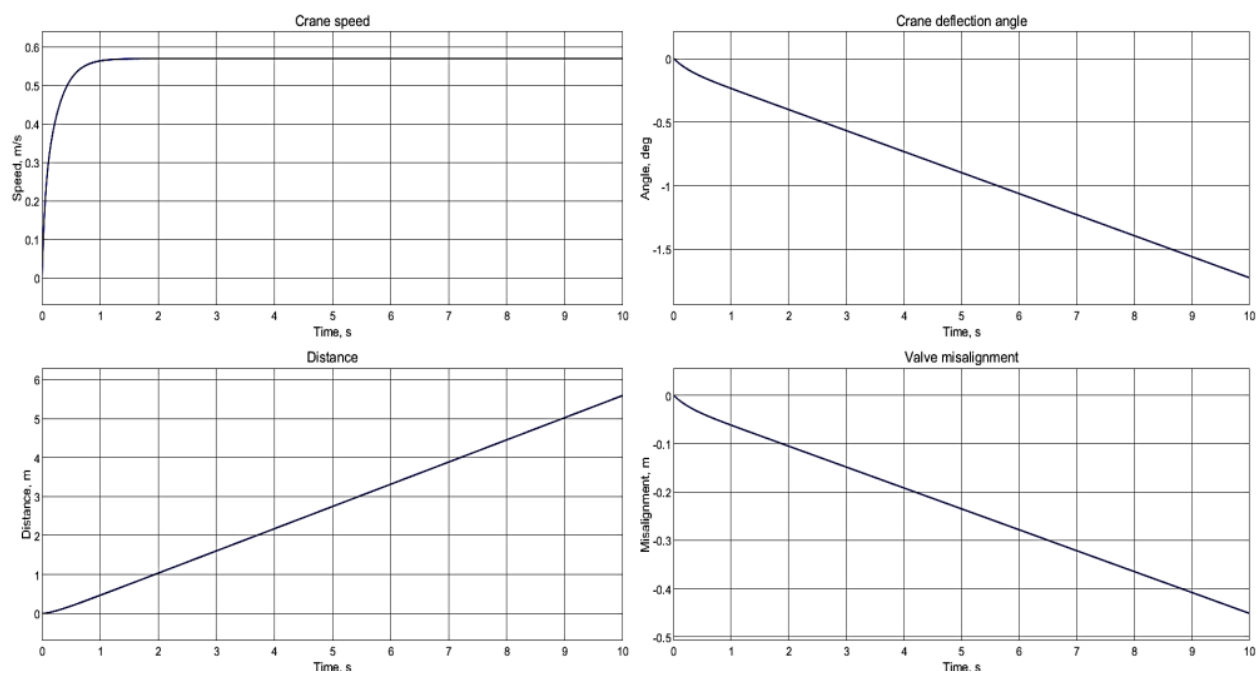

Fig. 13. Graphs of model parameters with shifted load.

Fig. 11 shows a graph of the drive transition process, the model of which is shown in Fig. 4. The desired speed is set to $0.6 \mathrm{~m} / \mathrm{s}$. This value is the maximum speed for the crane model under study. There is no load on the drive, and it reaches the set speed of rotation.

To confirm the adequacy of the model and its compliance with the specified requirements, a number of experiments were conducted. Fig. 12 and 13 show the readings of the graphs of the model with the position of the load in the center of the crane bridge and shifted to the right side of the direction of movement, respectively.

According to the graphs, it can be concluded that the model describes the movement and misalignment of the crane. There is no need to limit the misalignment angle, because the control system must minimize the angle and it must not reach values that are unacceptable from the point of view of the geometry of the structure.

Fig. 14 and 15 show the graphs of transition processes with the misalignment compensation system turned off and on. The tests were carried out on the model shown in Figure 10. In this case, a load of 16,000 kilograms is located in the middle of the right half of the crane bridge. The second graph is scaled to demonstrate the transition process in the amount of the crane misalignment.

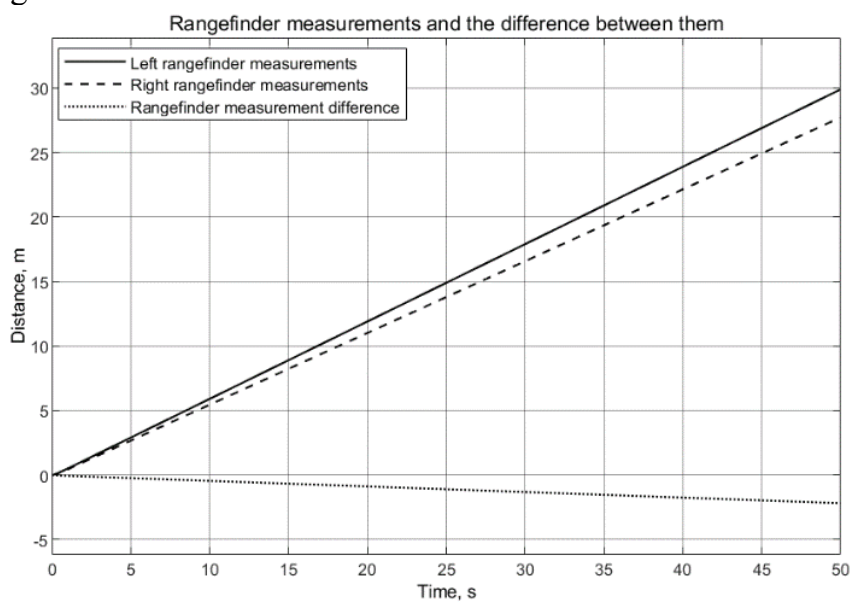

Fig. 14. Graph of rangefinder readings with the misalignment compensation system turned off. 


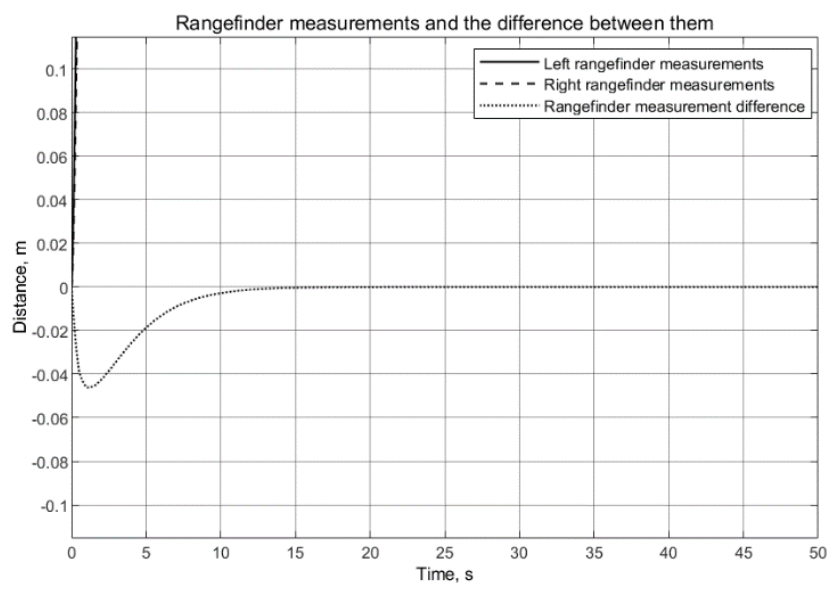

Fig. 14. Graph of rangefinder readings with the misalignment compensation system turned on

According to the graphs showing one of the many experiments performed, it can be judged that such a model is adequate. The amount of misalignment reaches $45 \mathrm{~mm}$, which can be considered acceptable, because with such geometrical characteristics of the crane and wheels, the misalignment value at which the wheel flanges will not touch the rails can reach $100 \mathrm{~mm}$.

\section{Conclusion}

As a result of this work, a mathematical model was obtained that describes the process of misalignment of the load-bearing beam of an overhead crane. Based on the obtained mathematical model, a model was implemented in Matlab Simulink, which reproduced the misalignment of the crane depending on the specified parameters. Then a control system was implemented and simulated, which included two laser rangefinders as sensors that measure the amount of misalignment, and a crane control block that corrects the speeds of the drives and thereby minimizes the amount of misalignment of the crane.

Through a series of experiments, it was revealed that the developed control system copes with the task of countering the crane misalignment. In the further development of the work, it is planned to improve the crane model and control system in order to bring the processes closer to real ones.

\section{References}

1. A.E. Komarov, A.A. Grachev, A.S. Gabriel, Analysis of the possibility of installation of the underway crane crane beam control, JARiTS 20, (2020)

2. Official catalog of models of overhead cranes of the Stahl Crane Systems company [Electronic resource]. https://stahlnw.ru/wp-content/uploads/docs/brochures/kranovyesistemy-STAHL.pdf

3. V.P. Zhegulsky, O.A. Lukashuk, Design, construction and calculation of bridge crane mechanisms: a tutorial.184 p. (2016)

4. S. Sitthipong et al., Design analysis of overhead crane for maintenance workshop, MATEC Web Conf. 207, 1-5 (2018)

5. A. Krishan, Damage of Bridge Lifting Cranes and Crane Metal Structures, Journal of Engineering and Applied Sciences 12(23), (2017)

6. L. Sowa, P. Kwiatoń, Mathematical modeling of mechanical phenomena in the gantry crane 
beam, Journal of Applied Mathematics and Computational Mechanics 16(3), 97-104 (2017)

7. Z. Zhang, D. Chen, M. Feng, Dynamics model and dynamic simulation of overhead crane load swing systems based on the ADAMS, $9^{\text {th }}$ Int. Conf. Comput. Ind. Des. Concept. Des. Multicult. Creat. Des. pp. 484-487 (2008)

8. A.P. Kuznetsov, A.V. Markov, A.S. Shmarlovsky, Mathematical models of gantry cranes 46, 93-100 (2009)

9. R.M.T.R. Ismail et al., Nonlinear dynamic modelling and analysis of a 3-D overhead gantry crane system with payload variation, EMS 2009 - UKSim 3rd Eur. Model. Symp. Comput. Model. Simul. pp. 350-354 (2009)

10. A.M. Bukhlakov, S.E. Gilev, A.M. Zyuzev, Imitation model of the electric drive of the bridge crane carrier. pp. 326-329 (2017)

11. The official catalog of models of wheel blocks of the Stahl Crane Systems company https://d1dv5w06e8cxfl.cloudfront.net/fileadmin/user_upload/Dokumente/Drucke/Prod uktinfos/Pi_Radblock_2019-01.pdf (date accessed: 04/26/2021).

12. V.V. Karzhavin, S.F. Kamenskikh, I.V. Dushanin, Calculation of crane mechanisms. Course project (2013)

13. J. Ryde, N. Hillier, Performance of laser and radar ranging devices in adverse environmental conditions, J. F. Robot. 26, 712-727 (2009)

14. O. Formsma et al., Realistic simulation of laser range finder behavior in a smoky environment, Lect. Notes Comput. Sci. 6556, 336-349 (2011)

15. J.F. Li et al., A novel positioning system of overhead crane, Int. Conf. Electr. Control Eng. ICECE. pp. 209-212 (2010) 